Boise State University

ScholarWorks

4-2-2009

\title{
The Virtual Refraction: Useful Spurious Energy in Seismic Interferometry
}

Dylan Mikesell

Boise State University

Kasper van Wijk

Boise State University

Alexander Calvert

ION-GX Technology

Matthew M. Haney

Boise State University 


\title{
The virtual refraction: Useful spurious energy in seismic interferometry
}

\author{
Dylan Mikesell ${ }^{1}$, Kasper van Wijk ${ }^{1}$, Alexander Calvert ${ }^{2}$, and Matt Haney ${ }^{3}$
}

\begin{abstract}
Seismic interferometry is rapidly becoming an established technique to recover the Green's function between receivers, but practical limitations in the source-energy distribution inevitably lead to spurious energy in the results. Instead of attempting to suppress all such energy, we use a spurious wave associated with the crosscorrelation of refracted energy at both receivers to infer estimates of subsurface parameters. We named this spurious event the virtual refraction. Illustrated by a numerical two-layer example, we found that the slope of the virtual refraction defines the velocity of the faster medium and that the stationary-phase point in the correlation gather provides the critical offset. With the associated critical time derived from the real shot record, this approach includes all of the necessary information to estimate wave speeds and interface depth without the need of inferences from other wave types.
\end{abstract}

\section{INTRODUCTION}

The Green's function between two receivers is obtained by crosscorrelating the recorded wavefields at the receivers from sources located everywhere in the media (Lobkis and Weaver, 2001; Weaver and Lobkis, 2001; Derode et al., 2003; Roux and Fink, 2003; Snieder, 2004; Roux et al., 2005). Shapiro and Campillo (2004) and Sabra et al. (2005) use ocean noise to recover the surface-wave part of the elastic Green's function; Malcolm et al. (2004) use the seismic coda to estimate the surface-wave Green's function between receivers. Wapenaar and Fokkema (2006) and van Manen et al. (2005) show that in media without attenuation, recovery of the Green's function is exact when monopole and dipole sources surround the receivers on a closed surface. In real data, these conditions cannot be met, although even with limited source distributions it is possible to extract important medium parameters (Schuster et al., 2004; Bakulin and Calvert, 2006; van Wijk, 2006). Dong et al. (2006) use refracted energy in seismic interferometry to distinguish between head waves and diving waves.

In this work, we explain the origin of spurious energy related to refracted waves. More importantly, we use this spurious event, which we call the virtual refraction, to quantify subsurface parameters (i.e., wave speeds and depth to interface). Using numerical examples, we show the cause of this spurious wave. We illustrate its use in a numerical two-layer refraction experiment, providing an alternative approach to conventional intercept-time seismic refraction analysis (Palmer, 1986; Lowrie, 2007). Finally, we discuss possible advantages of this technique in conventional refraction methods - particularly the ease of picking the virtual refraction velocity (even in the presence of noise) and its self-contained nature to invert for subsurface parameters.

\section{MODEL AND INTERFEROMETRIC RESULT}

Consider the two-layer acoustic model shown in Figure 1. The top layer has velocity $V_{0}=1250 \mathrm{~m} / \mathrm{s}$, the bottom layer has velocity $V_{1}$ $=1750 \mathrm{~m} / \mathrm{s}$, and the density is constant within the model. We place an explosive seismic source (with a dominant wavelength of approximately $30 \mathrm{~m}$ ) at the first receiver location $r 1$ and model the wavefield for $0.8 \mathrm{~s}$ after the explosion on 101 receivers, evenly spaced on a 400-m line located $52 \mathrm{~m}$ above the interface.

We use the spectral element modeling method, widely used in global seismology (Komatitsch and Vilotte, 1998; Komatitsch and Tromp, 2002). Figure $2 \mathrm{a}$ shows three coherent events in the modeled wavefield: the direct wave, the wave reflected from the interface, and the refracted wave at offsets greater than $300 \mathrm{~m}$. Using seismic interferometry, we attempt to recover the wavefield between two receiver positions based on Wapenaar and Fokkema's (2006) equation 19, which represents the exact acoustic Green's function:

Manuscript received by the Editor 4 September 2008; revised manuscript received 21 November 2008; published online 2 April 2009.

${ }^{1}$ Boise State University, Physical Acoustics Lab and Department of Geosciences, Boise, Idaho, USA. E-mail: dmikesell@cgiss.boisestate.edu; kasper @ cgiss.boisestate.edu.

ION-GX Technology, Denver, Colorado, USA.E-mail: alex.calvert@iongeo.com.

${ }^{3}$ U.S.G.S. Alaska Volcano Observatory, Anchorage, Alaska, USA. E-mail: mhaney@usgs.gov. (C) 2009 Society of Exploration Geophysicists. All rights reserved. 


$$
\begin{aligned}
\hat{G}\left(\mathbf{x}_{A}, \mathbf{x}_{B}, \omega\right)+\hat{G}^{*}\left(\mathbf{x}_{A}, \mathbf{x}_{B}, \omega\right) & \\
= & \oint_{S} \frac{-1}{j \omega \rho(\mathbf{x})}\left(\hat{G}^{*}\left(\mathbf{x}_{A}, \mathbf{x}, \omega\right) \partial_{i}\left(\hat{G}\left(\mathbf{x}_{B}, \mathbf{x}, \omega\right)\right)\right. \\
& \left.-\partial_{i}\left(\hat{G}^{*}\left(\mathbf{x}_{A}, \mathbf{x}, \omega\right)\right) \hat{G}\left(\mathbf{x}_{B}, \mathbf{x}, \omega\right)\right) n_{i} d S
\end{aligned}
$$

where $\hat{G}\left(\mathbf{x}_{A}, \mathbf{x}_{B}, \omega\right)$ denotes the causal frequency-domain Green's function at $\mathbf{x}_{A}$ from a source at $\mathbf{x}_{B}$ and where $\hat{G}^{*}\left(\mathbf{x}_{A}, \mathbf{x}_{B}, \omega\right)$ denotes the complex conjugate Green's function, which corresponds to the anticausal time-domain Green's function. The values $\hat{G}^{*}\left(\mathbf{x}_{A}, \mathbf{x}, \omega\right)$ and $\hat{G}\left(\mathbf{x}_{B}, \mathbf{x}, \omega\right)$ represent the Green's functions at locations $\mathbf{x}_{A}$ and $\mathbf{x}_{B}$ resulting from a monopole source at $\mathbf{x} ; \partial_{i} \hat{G}^{*}\left(\mathbf{x}_{A}, \mathbf{x}, \omega\right)$ and $\partial_{i} \hat{G}\left(\mathbf{x}_{B}, \mathbf{x}, \omega\right)$ represent the Green's functions at locations $\mathbf{x}_{A}$ and $\mathbf{x}_{B}$ resulting from a dipole source at $\mathbf{x}$. The value $S$ is the closed integration surface around the receivers $\mathbf{x}_{A, B}$.

To approximate this analytic result, we use a finite number of sources on a surface surrounding our receivers (Figure 1). We choose the integration surface $S$ to be a circle with a radius of $475 \mathrm{~m}$. We place the receiver array $75 \mathrm{~m}$ to the right of the circle's center. Then we distribute 2880 dipole and monopole seismic sources evenly over the circle, approximately one dipole and monopole source every meter along the circle. We simulate a monopole source using an explosive source. A dipole source consists of the sum of an explosion (located $2.5 \mathrm{~m}$ outside the circle) and an implosion (located $2.5 \mathrm{~m}$ inside the circle), divided over the distance between them $(5 \mathrm{~m})$. We center the dipole at the monopole source location and orient the dipole normal to the circle.

Next, we crosscorrelate the wavefields according to equation 1 for each source position. We set $\mathbf{x}_{B}=r 1$ so that it is always the receiver crosscorrelated with the 101 receivers ( $r 1$ is commonly called the virtual shot location; Bakulin and Calvert [2004]). After summing the crosscorrelations for all sources, we obtain the virtual shot record shown in Figure 2b. Because we are limited to a finite number of sources on $S$, we observe some noise before the first breaks. There is a phase difference in the source wavelet between the

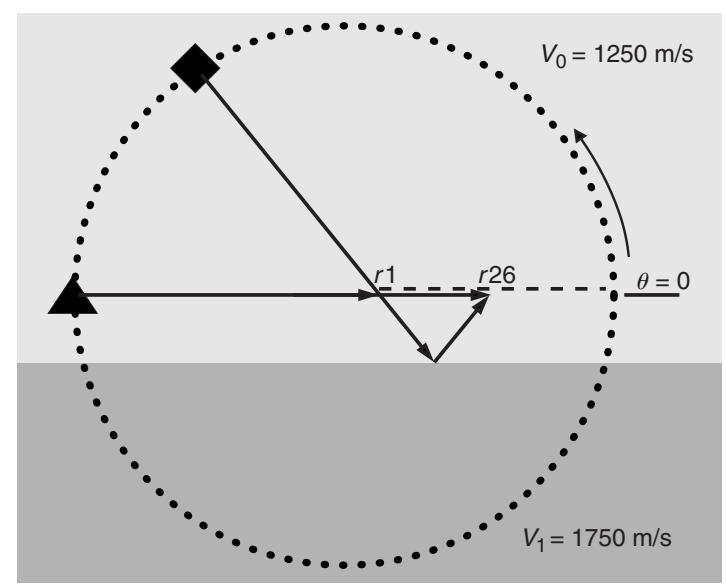

Figure 1. Layout of the acoustic numeric model with 2880 sources on a circle with radius of $475 \mathrm{~m}$ and 101 receivers every $4 \mathrm{~m}$ on the dashed line, $52 \mathrm{~m}$ above the interface. Receiver $r 1$ is located $75 \mathrm{~m}$ to the right of the circle center. The diamond and square infer stationary-phase points. real and the virtual shot records because the source wavelet is squared in the crosscorrelation procedure of seismic interferometry (Snieder, 2004; Wapenaar and Fokkema, 2006).
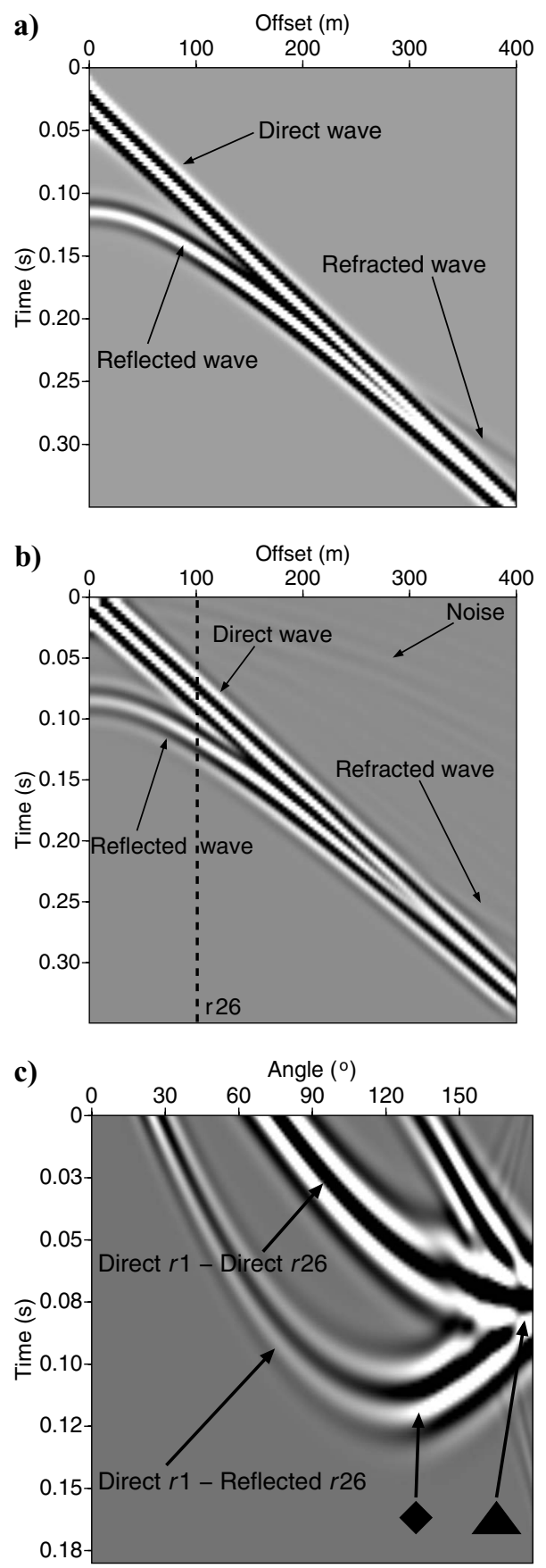

Figure 2. (a) Shot record from an explosive source placed at receiver $r 1$ (i.e., zero offset), showing the direct, reflected, and refracted waves. (b) Virtual shot record based on a discretized equation 1. The wavelet in the seismic interferometry result is the autocorrelation of the real shot wavelet. (c) Correlation gather between $r 1$ and $r 26$ (dashed line, middle plot) for all monopole sources on the top half of the circle. Stationary-phase points are denoted with symbols corresponding to those in Figure 1. The triangle is related to the direct wave; the diamond is related to the reflected wave. 


\section{THE STATIONARY PHASE IN THE FAR-FIELD}

Wapenaar and Fokkema (2006) simplify equation 1 by making the following assumptions:

- All sources lie in the far-field (i.e., the distance from the source to the receivers and scatterers is large compared to the wavelength).

- Rays take off approximately normal from the integration surface $S$.

- The medium outside the integration surface $S$ is homogeneous, such that no energy going outward from the surface is scattered back into the system.

- The medium around the source is locally smooth (the high-frequency approximation).

Following these assumptions, the spatial derivative can be approximated by a time derivative:

$$
\partial_{i} \hat{G}\left(\mathbf{x}_{A}, \mathbf{x}, \omega\right) n_{i} \approx-j \frac{\omega}{c(\mathbf{x})} \hat{G}\left(\mathbf{x}_{A}, \mathbf{x}, \omega\right) .
$$

This simplifies our equation 1 to Wapenaar and Fokkema's (2006) equation 31 :

$$
\begin{aligned}
\hat{G}\left(\mathbf{x}_{A}, \mathbf{x}_{B}, \omega\right)+\hat{G}^{*}\left(\mathbf{x}_{A}, \mathbf{x}_{B}, \omega\right) \\
\quad \approx \oint_{S} \frac{2 \hat{G}^{*}\left(\mathbf{x}_{A}, \mathbf{x}, \omega\right) \hat{G}\left(\mathbf{x}_{B}, \mathbf{x}, \omega\right)}{\rho(\mathbf{x}) c(\mathbf{x})} d S .
\end{aligned}
$$

From this expression, we investigate the origin of the events in the virtual shot record using the stationary-phase argument (e.g., Snieder, 2004). Figure $2 \mathrm{c}$ presents the causal part of the correlations between $r 1$ and $r 26$ (i.e., offset equals $100 \mathrm{~m}$ ) for all monopole sources in the upper hemisphere of integration surface $S$ to illustrate the validity of the far-field approximation for the direct and reflected waves. We ignore correlations from sources in the lower half because no stationary points exist.

We observe several coherent events in this so-called correlation gather that result in the events in the virtual shot record (Mehta et al., 2008). The correlation of the direct wave at $r 1$ with the direct wave at $r 26$ has a stationary-phase point at $180^{\circ}$ and is associated with the direct wave traveling from $r 1$ to $r 26$ in approximately $0.08 \mathrm{~s}$. The arrows marked with a black triangle in Figures 1 and $2 \mathrm{c}$ indicate that this is the source location where source and receiver are inline.

Another coherent event in the virtual shot record stems from the correlation between the direct wave at receiver $r 1$ and the reflected wave at $r 26$. This event has a stationary-phase point at approximately $120^{\circ}$ associated with the reflected wave traveling from $r 1$ to $r 26$ in about $0.12 \mathrm{~s}$. The arrows marked with a black diamond in Figures 1 and $2 \mathrm{c}$ indicate that this is the source location where the wave reflects to $r 26$ after passing through $r 1$. These stationary-phase points result in the two arrivals in Figure $2 \mathrm{~b}$ at $100 \mathrm{~m}$ offset. The weaker correlations associated with refractions from sources past $150^{\circ}$ are discussed next.

\section{VIOLATION OF THE FAR-FIELD APPROXIMATION}

Figure 3a is a magnification of the weaker correlations in Figure 2c, except this time we show the correlations between receivers $r 1$ and $r 101$ to emphasize the longer offsets. These are correlations between the refracted wave at $r 101$ and either the direct, reflected, or refracted wave at $r 1$. For this particular model, sources between about $170^{\circ}$ and the layer interface provide the visible stationary phase in the correlation at about $0.23 \mathrm{~s}$. This correlation is between the refracted wave at $r 1$ and the refracted wave at $r 101$ (see Figure $3 b)$. These refractions have paths in common to and from the refractor. Hence, the time of the correlation is only a function of the difference in travel paths along the refractor, denoted by $d r$ in Figure $3 b$.

This correlation does not cancel when summing over monopole sources only. Figure $4 \mathrm{a}$ is the virtual shot record using the approximate far-field result of equation 3 (i.e., using only monopole sources on the circle). Again, we recover the correct direct, reflected, and refracted waves. However, we also observe a spurious linear event traveling at $V_{1}=1750 \mathrm{~m} / \mathrm{s}$ going through the origin. This spurious wave is the virtual refraction. We deduce this event is a direct result of violating the far-field approximation represented by equation 2 , because it is the only approximation we made to the exact interferometric result of equation 1, depicted in Figure 2b. Equation 2 is incorrect for those sources where the interface between $V_{0}$ and $V_{1}$ is located in the near-field. Figure $3 b$ identifies these sources, which generate refracted energy.

\section{A LINE OF SOURCES}

Sources located at postcritical locations on the circle are responsible for the virtual refraction, therefore, we can instead place explosive sources on a line beyond the critical angle to enhance the event. Figure $3 b$ illustrates how postcritical sources on the circle can be transposed onto such a line.

We place a line of 110 explosive sources $52 \mathrm{~m}$ above the interface, to the left of the receiver line. This model closely resembles a common 2D data acquisition geometry in seismic surveys, except that we more commonly perform this experiment at the surface. Here, we bury sources and receivers to eliminate correlations associated with
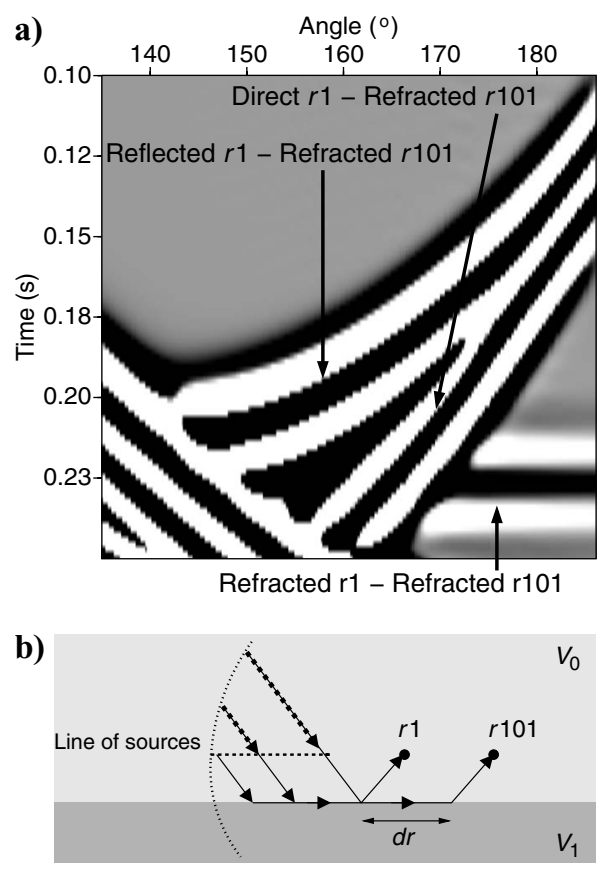

Figure 3. (a) Correlation gather between $r 1$ and $r 101$. (b) Paths for refracted waves traveling from the source to $r 1$ and $r 101$. Postcritical sources on the circle can be transposed to a line of sources. 
surface waves and surface-related multiples. Figure $4 \mathrm{~b}$ shows the virtual shot record obtained following equation 3 and the line of explosive sources. We identify the direct, reflected, and refracted waves as well as the virtual refraction.
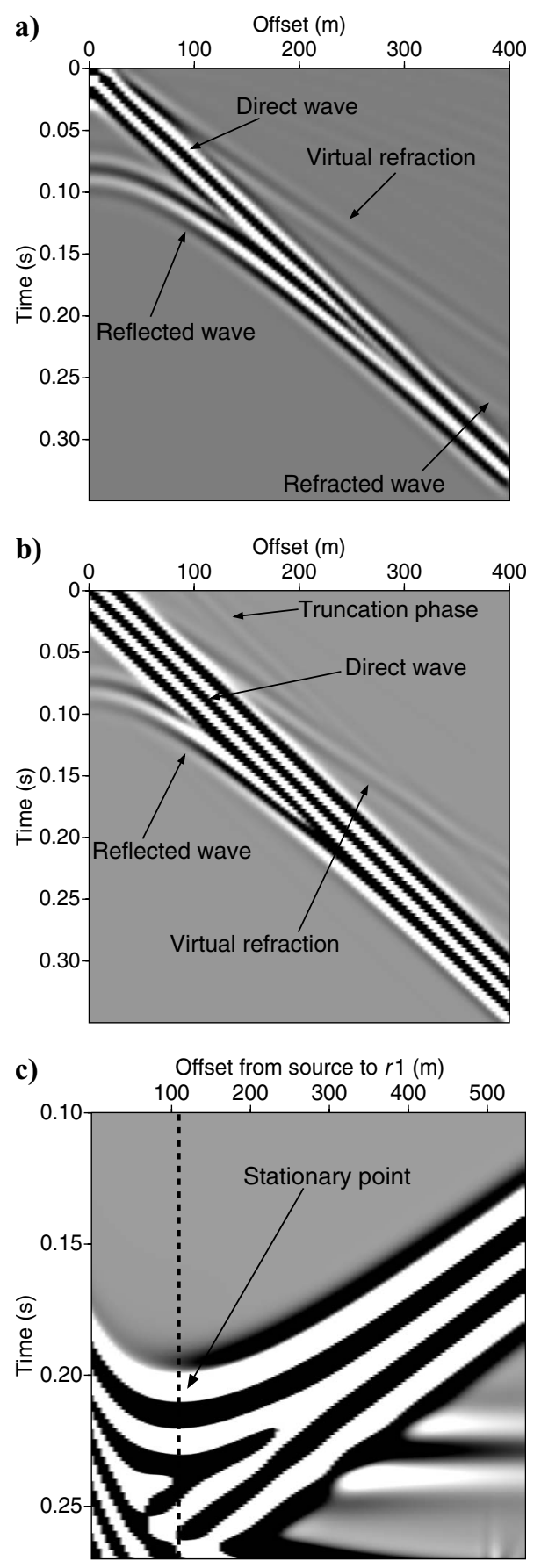

Figure 4. (a) The virtual shot record using only explosive sources on the circle. In addition to the direct, reflected, and refracted waves, we observe a linear spurious event: the virtual refraction. (b) The virtual shot record for a line of explosive sources, showing direct and reflected waves, along with a strong virtual refraction. (c) The correlation gather for $r 1$ and $r 101$ with a constant phase for the correlation between refracted waves at the larger offsets. Note the stationaryphase point at the critical offset (dashed line) when the source is $106 \mathrm{~m}$ from $r 1$.
Although this virtual refraction is not part of the true Green's function, its moveout defines the wave speed $V_{1}$ in the bottom layer. In the presence of a dipping refractor, one would perform seismic interferometry with source lines on both sides of the receiver line. The average of the two speeds observed in the virtual refractions would determine $V_{1}$, similar to conventional refraction techniques (Palmer, 1986). Other linear events not crossing the origin are truncation phases, because our source coverage is abruptly ended on each side of the source line (Snieder et al., 2008). Because all contributions from postcritical sources sum constructively, the virtual refraction is robust in the presence of uncorrelated noise.

\section{The critical offset}

By definition, the intercept time of the virtual refraction is $t=0 \mathrm{~s}$. Therefore, unlike in conventional refraction analysis (Palmer, 1986; Lowrie, 2007), important subsurface information about the top-layer velocity $V_{0}$ and interface depth $H$ cannot be determined from the virtual shot record alone.

Let us examine the correlation gather between $r 1$ and $r 101$ for the line of explosive sources (Figure 4c). For long-offset sources, we see the constant feature at $t \approx 0.23 \mathrm{~s}$. The correlation between the direct and refracted waves is represented by a straight line; the curving feature represents the correlation between the reflected and refracted waves, having an extremum at $x \approx 106 \mathrm{~m}$ (dashed line). This stationary-phase point associated with the correlation between reflected and refracted waves occurs at the critical offset. Using the sine of the critical angle $\sin \left(\theta_{c}\right) \equiv V_{0} / V_{1}$, Pythagorean theorem, and the parameters defined in Figure 5, we can write

$$
\sin \left(\theta_{c}\right) \equiv \frac{V_{0}}{V_{1}}=\frac{\frac{x_{c}}{2}}{\sqrt{\left(\frac{x_{c}}{2}\right)^{2}+H^{2}}} \Leftrightarrow x_{c}=\frac{2 V_{0} H}{\sqrt{V_{1}^{2}-V_{0}^{2}}} .
$$

To show that this is the same offset between the source and $r 1$ where the maximum delay between refracted and reflected waves occurs, we set the spatial derivative of the difference in arrival times to zero and solve for offset $x$ :

$$
\begin{aligned}
& \frac{d}{d x}\left(t_{\text {refr }}-t_{\text {reff }}\right)=0 \Leftrightarrow \\
& \frac{d}{d x}\left(\left(\frac{2 H \cos \theta_{c}}{V_{0}}+\frac{x}{V_{1}}\right)\right. \\
& \left.-\sqrt{\left(\frac{x}{V_{0}}\right)^{2}+\left(\frac{2 H}{V_{0}}\right)^{2}}\right)=0 \Leftrightarrow \\
& \frac{1}{V_{1}}-\frac{x}{V_{0} \sqrt{x^{2}+(2 H)^{2}}}=0 \Leftrightarrow x=\frac{2 V_{0} H}{\sqrt{V_{1}^{2}-V_{0}^{2}}},
\end{aligned}
$$

which equals the definition of the critical offset in equation 4 . The critical time $t_{c}$ can be picked on real data as the arrival time of the reflected event on $r 1$ for the source at $x_{c} \approx 106 \mathrm{~m}$. Then, with observables $x_{c}, t_{c}$, and $V_{1}$, we can solve for $H$ uniquely by rearranging equation 4: 


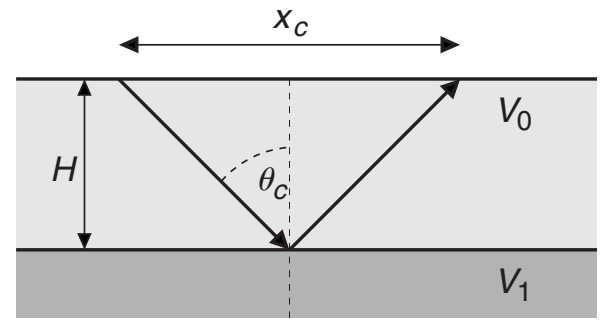

Figure 5. The raypath of reflection at the critical offset.

$$
H=\frac{x_{c} \sqrt{V_{1}^{2}-V_{0}^{2}}}{2 V_{0}} .
$$

Using $t_{c}=2 \sqrt{H^{2}+\left(x_{c} / 2\right)^{2}} / V_{0}$ and equation 6 , we can also solve for the top-layer wave speed $V_{0}$ :

$$
V_{0}=\sqrt{\frac{V_{1} x_{c}}{t_{c}}} .
$$

Although conventional refraction analysis in a horizontal twolayered model requires a separate estimate of $V_{0}$ (from the direct wave) to establish the top-layer thickness, in this analysis, refracted energy alone contains enough information to establish all of the unknowns without information from other wave types.

\section{CONCLUSIONS}

Spurious waves in practical applications of seismic interferometry are ever present because strict requirements for the full recovery of the Green's function between receivers cannot be met in practice. Here we present an artifact we call the virtual refraction in a two-layer model. We can estimate the velocity of the bottom layer from its slope and the critical offset from the stationary-phase point in the correlation gather between receivers. With the critical time picked on the real shot record, the real and virtual refractions provide enough information to estimate wave speeds and interface depth without information from other wave types. Finally, the virtual refraction intercepts at the origin and is the direct result of stacking multiple sources. These characteristics potentially provide robustness in the presence of noise.

\section{ACKNOWLEDGMENTS}

We are grateful for the critical and constructive comments of Kurang Mehta, Jan Thorbecke, Ludmila Adam, Joel Brown, and one anonymous reviewer. We would like to thank Dimitri Komatitsch for providing — and teaching us how to use — the spectral element code. We acknowledge NDSEG for supporting Dylan Mikesell and ION-GX Technology for supporting the Physical Acoustics Lab.

\section{REFERENCES}

Bakulin, A., and R. Calvert, 2004, Virtual source: New method for imaging and 4D below complex overburden: 74th Annual International Meeting, SEG, Expanded Abstracts, 2477-2480.

, 2006, The virtual source method: Theory and case study: Geophysics, 71, no. 4, SI139-SI150.

Derode, A., E. Larose, M. Campillo, and M. Fink, 2003, How to estimate the Green's function of a heterogeneous medium between two passive sensors? Application to acoustic waves: Applied Physics Letters, 83, 3054-3056.

Dong, S., J. Sheng, and J. T. Schuster, 2006, Theory and practice of refraction interferometry: 76th Annual International Meeting, SEG, Expanded Abstracts, 3021-3025.

Komatitsch, D., and J. Tromp, 2002, Spectral-element simulations of global seismic wave propagation - I. Validation: Geophysical Journal International, 149, 390-412.

Komatitsch, D., and J.-P. Vilotte, 1998, The spectral element method: An efficient tool to simulate the seismic response of 2D and 3D geological structures: Bulletin of the Seismological Society of America, 88, 368-392.

Lobkis, O. I., and R. L. Weaver, 2001, On the emergence of the Green's function in the correlations of a diffuse field: Journal of the Acoustical Society of America, 110, 3011-3017.

Lowrie, W., 2007, Fundamentals of geophysics, 2nd ed.: Cambridge University Press.

Malcolm, A. E., J. A. Scales, and B. A. van Tiggelen, 2004, Extracting the Green function from diffuse, equipartitioned waves: Physical Review E, 70, 015601 .

Mehta, K., R. Snieder, R. Calvert, and J. Sheiman, 2008, Acquisition geometry requirements for generating virtual-source data: The Leading Edge, 27 620-629.

Palmer, D., 1986, Refraction seismics: The lateral resolution of structure and seismic velocity: Geophysical Press.

Roux, P., and M. Fink, 2003, Green's function estimation using secondary sources in a shallow water environment: Journal of the Acoustical Society of America, 113, 1406-1416.

Roux, P., K. Sabra, W. A. Kuperman, and A. Roux, 2005, Ambient noise cross correlation in free space: Theoretical approach: Journal of the Acoustical Society of America, 117, 79-84.

Sabra, K. G., P. Gerstoft, P. Roux, W. A. Kuperman, and M. C. Fehler, 2005, Extracting time-domain Green's function estimates from ambient seismic noise: Geophysical Research Letters, 32, L03310.

Schuster, G. T., J. Yu, J. Sheng, and J. Rickett, 2004, Interferometric/daylight seismic imaging: Geophysical Journal International, 157, 838-852.

Shapiro, N. M., and M. Campillo, 2004, Emergence of broadband Rayleigh waves from correlations of the ambient seismic noise: Geophysical Research Letters, 31, L07614.

Snieder, R., 2004, Extracting the Green's function from the correlation of coda waves: A derivation based on stationary phase: Physical Review E, 69, 046610

Snieder, R., K. van Wijk, M. Haney, and R. Calvert, 2008, Cancellation of spurious arrivals in Green's function extraction and the generalized optical theorem: Physical Review E, 78, 036606.

van Manen, D.-J., J. O. A. Robertsson, and A. Curtis, 2005, Modeling of wave propagation in inhomogeneous media: Physical Review Letters, 94 164301

van Wijk, K., 2006, On estimating the impulse response between receivers in a controlled ultrasonic experiment: Geophysics, 71, no. 4, S179-S184.

Wapenaar, K., and J. Fokkema, 2006, Green's function representations for seismic interferometry: Geophysics, 71, no. 4, S133-S146.

Weaver, R. L., and O. I. Lobkis, 2001, Ultrasonics without a source: Thermal fluctuation correlations at MHz frequencies: Physical Review Letters, 87, 134301 